\title{
Trimethylsilyl Cyanide (TMSCN)
}

\section{Laura L. Romero-Hernández}

Facultad de Ciencias Químicas, Benemérita Universidad Autónoma de Puebla, Ciudad Universitaria, Av. San Claudio y Blvd. 14 sur. Puebla, Puebla C.P. 72570, Mexico laura.romerohernandez@correo.buap.mx

Published online: 06.02 .2015

DOI: 10.1055/s-0034-1380231; Art ID: st-2014-v0510-v
Laura L. Romero-Hernández was born in 1988 in Puebla, Mexico. She obtained her B.Sc. degree in Chemistry (2011) and her M.Sc. degree in organic chemistry (2014) from Benemérita Universidad Autónoma de Puebla. Currently she works towards her Ph.D. under the guidance of Professor Sara Montiel Smith and Professor Penélope Merino Montiel. Her research focuses on the synthesis of steroidal spiro heterocycles.

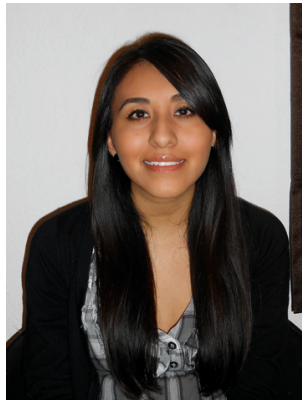

a)

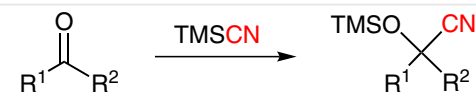

ketone or aldehyde

b)

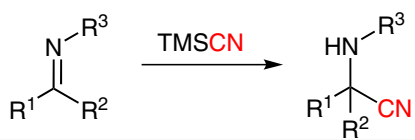

Scheme 1 Main uses of TMSCN reactions, but it is generally used in nucleophilic additions to aldehydes, ketones and imines to form cyanohydrin silyl ethers ${ }^{2}$ (Scheme 1a) and $\alpha$-aminonitriles ${ }^{3}$ in Strecker-type reactions (Scheme $1 \mathrm{~b})$.

Table 1 Use of TMSCN

(A) The direct substitution of benzylic and allylic alcohols by cyanide using TMSCN and Brønsted acid Montmorillonite catalysts was developed by Wang et al. After optimizing the reaction conditions, the compounds were obtained with moderate to excellent yields and high regioselectivity. ${ }^{4}$

(B) The thiocyanate group is a useful moiety for the synthesis of various heterocycles ${ }^{5}$ and it is an important function which has shown biological activity in a series of derivatives. ${ }^{6}$ Recently, Yamaguchi et al. reported the synthesis of alkyl and aryl thiocyanates by nucleophilic cyanation of thiols using TMSCN, manganese oxide octahedral molecular sieves (OMS-2), $\mathrm{KF}$ and $\mathrm{O}_{2}$ to afford the products with excellent yields $(>90 \%)^{7}$

(C) A novel stereospecific synthesis of acyl cyanides from propargyl aldehydes was described by Choi et al. The procedure involves the use of $\mathrm{Et}_{3} \mathrm{~N}$ as dual catalyst, acting as a Lewis base in the cyanosilylation process and then as a Brønsted base in the umpolung step. The final hydrolysis and tautomerization of the resulting allene gave the (E)-isomers with moderate to good yields. ${ }^{8}$

(D) Arai et al. reported the dicyanation of alkynes using TMSCN, $\mathrm{Pd}(\mathrm{CN})_{2}$ as the catalyst and atmospheric $\mathrm{O}_{2}$. The mechanism begins with the $\pi$-complex formation and subsequent nucleophilic attack of the cyano group (syn or anti) to one carbon of the alkyne. The reductive elimination afforded the dicyanated products with good diastereoisomeric excess depending on the substrates. ${ }^{9}$

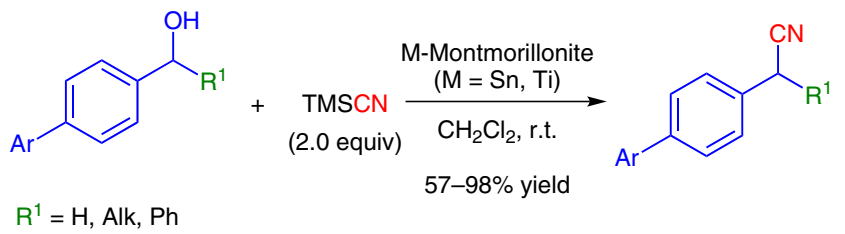

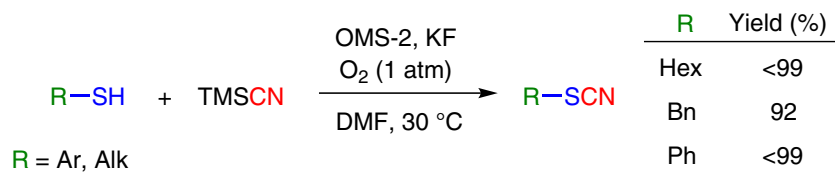

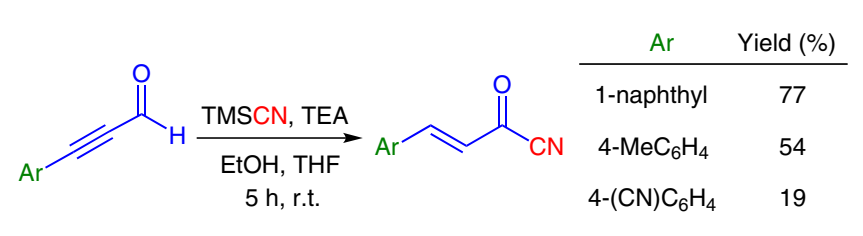

$$
\mathrm{R}^{1}=\mathrm{R}^{2} \underset{\mathrm{PhMe}, 100^{\circ} \mathrm{C}}{\stackrel{\mathrm{O}_{2}(1 \mathrm{~atm})}{\mathrm{TMSCN} \mathrm{PdX}_{2}(\mathrm{X}=\mathrm{Cl}, \mathrm{CN})}} \sum_{\mathrm{NC}}^{\mathrm{R}_{\mathrm{CN}}^{1}}
$$$$
\mathrm{R}^{1}=\mathrm{Alk}, \mathrm{Ph} \quad 45-86 \% \text { yield }
$$$$
\mathrm{R}^{2}=\mathrm{H}, \mathrm{Me}
$$ 
(E) A variety of aryl nitriles has been synthetized by Ye et al. by a cross-coupling reaction under mild and simple conditions. The reaction involves the use of aryl boronic acids or esters as starting materials, TMSCN as the cyanide source, $\mathrm{Cu}_{2} \mathrm{O}$ as the catalyst and DMEDA as a ligand under an $\mathrm{O}_{2}$ atmosphere. ${ }^{10}$

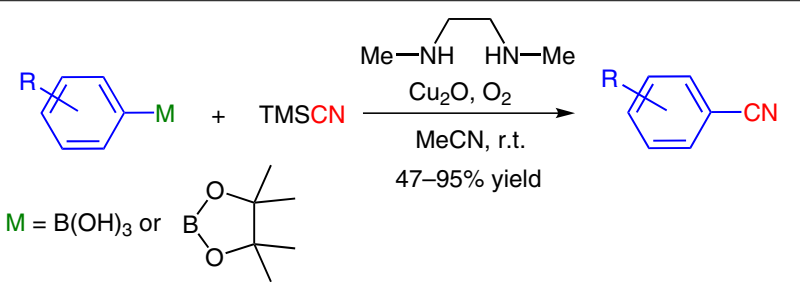

(F) TMSCN was used in the second step of an oxidative Michael addition of cyanide anion to Baylis-Hillman adducts. The importance of the addition of cyanide to $\alpha, \beta$-unsaturated carbonyl derivatives is that the products can be converted into a variety of compounds including $\gamma$-aminobutyric acids. The reaction took place in a liquid ionic medium ([bmim]Br), which was reused several times without losing its activity. The $\beta$-cyano carbonyl compounds were obtained with high regioselectivity and yields (>79\%). ${ }^{11}$

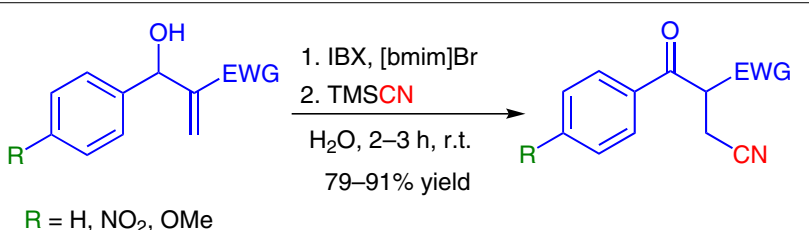

(G) Xu et al. described the $\alpha$-azidocyanation of aryl alkenes using TMSN $_{3}$ and TMSCN in the presence of an oxidative agent and a copper catalyst. The difunctionalization of alkenes with these versatile groups allows the transformation into many derivatives such as amino acids. The mechanism is believed to start by the formation of an azide radical that reacts with the alkene; the oxidation of the resulting radical leads to a carbocation which is finally trapped by the cyanide anion. The azidocyano derivatives were achieved with low to moderate yields depending on the substituents. ${ }^{12}$

$$
\mathrm{Ar}+\mathrm{TMSN}_{3}+\mathrm{TMSCN}_{\substack{\mathrm{MeOH} \text {, r.t. } \\ 32-84 \% \text { yield }}} \frac{\mathrm{Cu}(\mathrm{TFA})_{2}, \mathrm{Phl}(\mathrm{OAc})_{2}}{\longrightarrow}
$$

$(\mathrm{H})$ The regioselective cyanation of allylic derivatives was reported by Munemori et al. The best results were obtained using CuI, CuCN or $\mathrm{CuCl}_{2}$ as catalysts. The experiment in the presence of the free radical TEMPO showed that the cyanation proceeds through a radical pathway. The compounds were obtained with low to moderate yields. ${ }^{13}$

$\mathrm{R}^{1}=\mathrm{Ph}, \mathrm{Alk}$
$\mathrm{R}^{2}=\mathrm{H}, \mathrm{Me}, \mathrm{Ph}$
$\mathrm{X}=\mathrm{Cl}, \mathrm{Br}, \mathrm{OCO}_{2} \mathrm{Me}, \mathrm{OAc}$

\section{References}

(1) McBride, J. J. Jr; Beachell, H. C. J. Am. Chem. Soc. 1952, 74, 5247.

(2) Kikukawa, Y.; Suzuki, K.; Sugawa, M.; Hirano, T.; Kamata, K.; Yamaguchi, K.; Mizuno, N. Angew. Chem. Int. Ed. 2012, 51, 3686.

(3) Dekamin, M. G.; Mokhtari, Z. Tetrahedron 2012, 68, 922.

(4) Wang, J.; Masui, Y.; Onaka, M. ACS Catal. 2011, 1, 446.

(5) Langi, B. P.; Mulwad, V. V.; Chaskar, A. C. Acta Pol. Pharm. 2011, $68,441$.

(6) Silveira, G. P.; Ferreira, M.; Fernandes, L.; Moraski, G. C.; Cho, S.; Hwang, C.; Franzblau, S. G.; Sá, M. M. Bioorg. Med. Chem. Lett. 2012, 22, 6486

(7) Yamaguchi, K.; Sakagami, K.; Miyamoto, Y.; Jin, X.; Mizuno, N. Org. Biomol. Chem. 2014, DOI: 10.1039/C4OB01655F.
(8) Choi, H. H.; Son, Y. H.; Jung, M. S.; Kang, E. J. Tetrahedron Lett. 2011, 52, 2312.

(9) Arai, S.; Sato, T.; Koike, Y.; Hayashi, M.; Nishida, A. Angew. Chem. Int. Ed. 2009, 48, 4528.

(10) Ye, Y.; Wang, Y.; Liu, P.; Han, F. Chin. J. Chem. 2013, 31, 27.

(11) Yadav, L. D. S.; Awasthi, C.; Rai, A. Tetrahedron Lett. 2008, 49, 6360.

(12) Xu, L.; Mou, X.-Q.; Chen, Z.-M.; Wang, S.-H. Chem. Commun. 2014, 50, 10676.

(13) Munemori, D.; Tsuji, H.; Uchida, K.; Suzuki, T.; Isa, K.; Minakawa, M.; Kawatsura, M. Synthesis 2014, 46, 2747. 